\title{
Floating Stacking Fault during Homoepitaxial Growth of $\operatorname{Ag(111)~}$
}

\author{
S. A. de Vries, ${ }^{1}$ W. J. Huisman, ${ }^{1}$ P. Goedtkindt,,${ }^{1,2}$ M. J. Zwanenburg,,${ }^{1, *}$ S. L. Bennett,${ }^{3}$ and E. Vlieg ${ }^{1,4}$ \\ ${ }^{1}$ FOM-Institute for Atomic and Molecular Physics, Kruislaan 407, 1098 SJ Amsterdam, The Netherlands \\ ${ }^{2}$ Universitaire Instelling Antwerpen, Department of Chemistry, Universiteitsplein 1, B-2610 Wilrijk, Belgium \\ ${ }^{3}$ CCLRC, Daresbury Laboratory, Warrington WA4 4AD, United Kingdom \\ ${ }^{4}$ RIM, Department of Solid State Chemistry, University of Nijmegen, Toernooiveld 1, 6525 ED Nijmegen, The Netherlands
}

(Received 23 February 1998)

\begin{abstract}
We have investigated the influence of $\mathrm{Sb}$ on the formation of stacking faults during $\operatorname{Ag}(111)$ growth using x-ray scattering. In equilibrium, a predeposition of $\frac{1}{3}$ monolayer $\mathrm{Sb}$ results in a $(\sqrt{3} \times \sqrt{3}) R 30^{\circ}$ reconstruction in which the top layer is wrongly stacked. Upon continued $\mathrm{Ag}$ growth at $100{ }^{\circ} \mathrm{C}$, the $\mathrm{Sb}$ segregates and the $\mathrm{Ag}$ atoms return to the correct stacking, while the new $\mathrm{Ag}$ atoms in the top layer again have the wrong stacking. This thus effectively leads to a floating stacking fault. Because of kinetic limitations, the same effect occurs for lower Sb coverages. [S0031-9007(98)06618-6]
\end{abstract}

PACS numbers: 61.72.Nn, 61.10.-i, 68.55.-a

The growth of smooth and defect-free metallic layers is important in many technological areas, like metal-semiconductor contacts and magnetic multilayers. Usually, high substrate temperatures are required to grow smooth layers, but this may cause interdiffusion problems. It has been shown for a number of metals that homoepitaxial growth of smooth layers can be stimulated by using surfactants [1-4]. Surfactants have since also been used in the growth of metallic films on Si and sapphire [5-7].

The occurrence of stacking faults is an important factor for the quality of the layers, since it determines whether the film is continuous, twinned, or otherwise imperfect. At present, the influence of surfactants on the formation of stacking faults is not well understood. Submonolayer coverages of $\mathrm{Sb}$ change the growth mode of $\mathrm{Ag}(111)$ from multilayer (3D) growth to layer-by-layer (2D) growth [2], but this may sometimes enhance the formation of stacking faults [8]. On the other hand, the effect of $\mathrm{Pb}$ in the growth of $\mathrm{Cu} / \mathrm{Co}$ superlattices on $\mathrm{Cu}(111)[9,10]$ and the effect of In on the growth of $\mathrm{Cu}(111)$ [4] is to suppress the formation of stacking faults. For clean $\operatorname{Ag}(111)$ growth (without surfactant), Meinel, Klaua, and Bethge [11] observed $\operatorname{Ag}(111)$ growing with stacking faults at room temperature (RT) and that for 2D nucleation the probabilities for incorrect (hcp) and correct (fcc) atomic stacking are almost equal. Spot-profile analysis of low-energy electron diffraction experiments confirm this conclusion [12,13]. First principle calculations, on the other hand, show that for adatoms the fcc site is energetically slightly favored over the hcp site [14].

In this Letter we describe a remarkable, new effect of $\mathrm{Sb}$ during $\operatorname{Ag}(111)$ homoepitaxial growth. Sb causes the top surface layer to be at stacking fault positions, but during growth the surface unfaults so that the grown film is not faulted and the stacking fault floats along with the Sb.

We have used in situ surface $\mathrm{x}$-ray diffraction for this study. Sensitivity to the growth mode, surface roughness, the formation of stacking faults, and/or the pos- sible concomitant formation of twin crystallites is obtained by observing diffracted intensities at appropriate positions on so-called crystal truncation rods (CTRs) [15]. These CTRs are tails of diffuse intensity originating from the interference between bulk and surface atomic structure. These rods connect the bulk Bragg peaks in the direction perpendicular to the surface. Because of the weak interaction of $\mathrm{x}$ rays with matter the kinematical approximation is valid, which makes data analysis straightforward [16]. The large penetration depth makes it possible to study stacking fault and twin crystal formation at buried interfaces.

The measurements were performed at the surface x-ray diffraction station 9.4 of the wiggler beam line at the Synchrotron Radiation Source, Daresbury Laboratory, United Kingdom [17]. A wavelength of $0.9 \AA$ (13.8 keV) was selected using a channel-cut $\mathrm{Si}(111)$ monochromator. The setup consists of an ultrahigh-vacuum chamber [18] coupled to a diffractometer that was operated in six-circle mode [19]. The $\operatorname{Ag}(111)$ sample (miscut $\sim 0.2^{\circ}$ ) was cleaned by repeated cycles of sputtering $\left(600 \mathrm{eV} \mathrm{Ar}^{+}\right.$at $300{ }^{\circ} \mathrm{C}$ for $20 \mathrm{~min}$ ) and annealing $\left(600{ }^{\circ} \mathrm{C}\right.$ for $\left.10 \mathrm{~min}\right)$. Knudsen effusion cells were used for $\mathrm{Ag}$ and $\mathrm{Sb}$ deposition. The $\mathrm{Sb}$ deposition rate was $\sim 0.002$ monolayers (ML) per sec and the deposition rate of $\mathrm{Ag} \sim 0.01 \mathrm{ML}$ per sec. The deposited amounts of Sb given below have an error of $10 \%$.

For labeling the reflections we use a surface unit cell of which the primitive lattice vectors $\left\{\boldsymbol{a}_{i}\right\}$ can be expressed in the conventional bulk cubic fcc lattice vec-

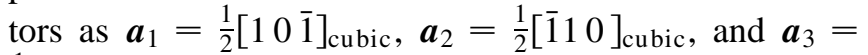
$\frac{1}{2}\left[\begin{array}{lll}1 & 1 & 1\end{array}\right]_{\text {cubic. }}$ The corresponding reciprocal lattice vectors $\left\{\boldsymbol{b}_{i}\right\}$ are defined by $\boldsymbol{a}_{i} \cdot \boldsymbol{b}_{j}=2 \pi \delta_{i j}$. The momentum transfer vector $\boldsymbol{Q}$, which is the difference between the wave vectors of the incident and scattered $\mathrm{x}$ rays, can be denoted by the diffraction indices $(h k \ell)$ in reciprocal space: $\boldsymbol{Q}=h \boldsymbol{b}_{1}+k \boldsymbol{b}_{2}+\ell \boldsymbol{b}_{3}$. For CTRs, which are labeled by $(h k)$, the indices $h$ and $k$ have integer values, whereas $\ell$ is 
unconstrained and refers to the component of $\boldsymbol{Q}$ perpendicular to the surface. The bulk Bragg peaks occur for integer values of $\ell$. The integrated intensity at each point $\ell$ is determined by rotating the crystal about the surface normal and measuring the number of diffracted photons. From the measured integrated intensities structure factors are derived in a standard fashion [20].

In order to investigate the formation of stacking faults during growth we have measured the $(h k)=(\overline{1} 0)$ CTR. The observed profiles for homoepitaxial growth of $\operatorname{Ag}(111)$ without the use of a surfactant are shown in Fig. 1 for three different temperatures. For each temperature, the structure factor amplitudes after growth are plotted as a function of $\ell$ (in reciprocal lattice units). Negative $\ell$ values are obtained by inverting the structure factor distribution along the positive $(h k)=(10)$ rod through the origin of reciprocal space (Friedel's rule). The expected structure factor distribution for the clean flat $\operatorname{Ag}(111)$ bulk terminated surface is given by the dashed curve. The bulk Bragg peaks are at $\ell=-1$ and $\ell=2$. At RT ( $3 \mathrm{ML} \mathrm{Ag} \mathrm{deposited)} \mathrm{and}-50{ }^{\circ} \mathrm{C}(4 \mathrm{ML}$ Ag deposited) twin crystallites are formed that are rotated $180^{\circ}$ with respect to the bulk. The twin Bragg peaks thus lie exactly on the bulk CTR, but at $\ell$ values that are the bulk values mirrored in the origin (i.e., at $\ell$ equals -2 and 1). The solid curves represent model calculations. At $-50{ }^{\circ} \mathrm{C}$ we find that $17 \%$ of the surface is covered by twins with a thickness of $4 \mathrm{ML}$. The RT data are best described by taking $8 \%$ of the surface to be covered by $3 \mathrm{ML}$ thick twin crystals. These twin crystals grow from a stacking fault formed at the annealed starting surface and then proceed with the "normal" fcc stacking $(A B C b a c b a c \ldots)$. Presumably, the stacking faults are

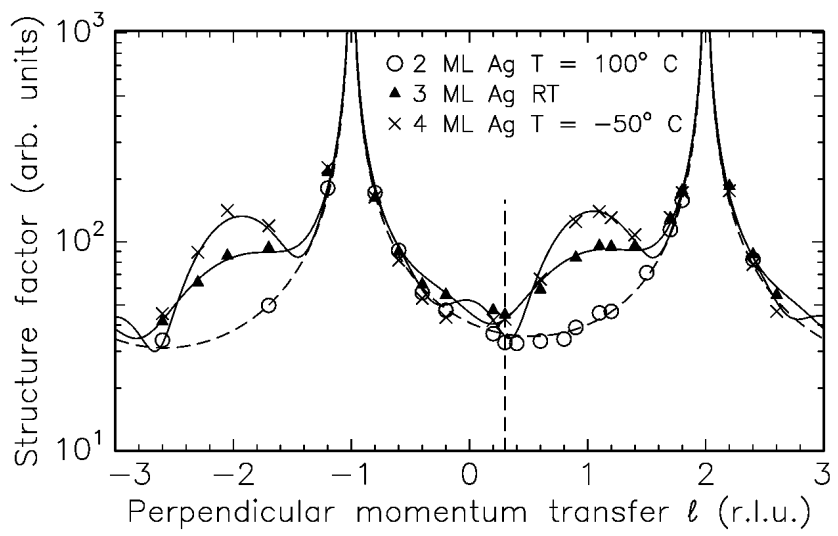

FIG. 1. Structure factors along the $(h k)=(\overline{1} 0)$ CTR after clean $\mathrm{Ag}$ growth (no predeposition of $\mathrm{Sb}$ ) at $-50^{\circ} \mathrm{C}, \mathrm{RT}$, and $100{ }^{\circ} \mathrm{C}$. The dashed curve gives the calculation for the bulk terminated $\operatorname{Ag}(111)$ surface and the solid curves represent best fits (see text). For the lower temperatures peaks start to appear at $l=-2$ and $l=1$ indicating twin crystal formation. The

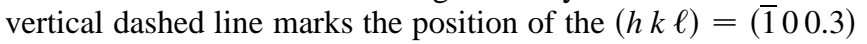
reflection which is monitored during deposition in Figs. 2 and 3 . formed at defects or impurities on the surface. After depositing the first layer these are covered and no new stacking faults are created. At $T=100{ }^{\circ} \mathrm{C}(2 \mathrm{ML} \mathrm{Ag}$ deposited) no twin crystal formation is observed and the data correspond to the calculation for the ideal, bulk terminated surface of $\operatorname{Ag}(111)$ [21]. We conclude that at higher temperatures fewer twin crystals are formed. This is caused by two effects. First, for higher temperatures, an increasing fraction of the arriving atoms will attach at step sites where they will reside at the correct stacking. Second, the higher mobility allows incorrectly nucleated islands to find the energetically favorable fcc site.

Before examining the effect of $\mathrm{Sb}$ on the formation of stacking faults, we first investigate $\mathrm{Sb}$ deposition on $\operatorname{Ag}(111)$ close to equilibrium. In Fig. 2 the intensity of the $(h k \ell)=(100.3)$ reflection is shown during $\mathrm{Sb}$ deposition at $250{ }^{\circ} \mathrm{C}$. This reflection is very sensitive to the occurrence of stacking faults, because atoms which grow on hcp sites interfere mainly constructively with the bulk, whereas atoms on the correct fcc sites interfere largely destructively. Note that this is different from specular reflectivity, where one is sensitive only to the out-of-plane position of the atoms, and not to their stacking [2]. Initially, the intensity remains constant, indicating that the surface remains smooth. Therefore, the $\mathrm{Sb}$ atoms must dissolve in the top layer, as was also found using scanning tunneling microscopy (STM) [22]. After deposition of approximately $\frac{1}{3}$ ML of Sb a sudden rise in intensity is observed which is indicative of the formation of stacking faults. The intensity increase coincides with the formation of a $(\sqrt{3} \times \sqrt{3}) R 30^{\circ}(\sqrt{3}$ for short) reconstruction, as was confirmed by measuring fractional order reflections. A detailed investigation of the exact atomic structure will be presented elsewhere [23], but in short we found that in equilibrium all top layer atoms are on stacking fault positions and each surface unit cell contains one substitutional $\mathrm{Sb}$ atom. This substitutional hcp site has not been considered in

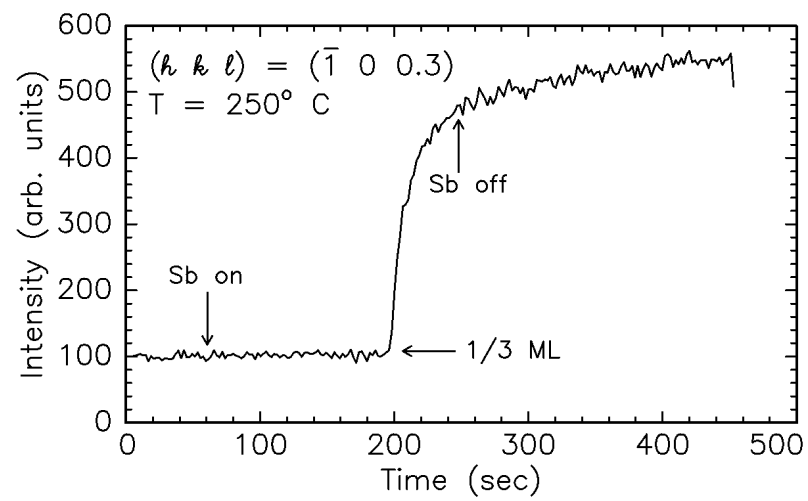

FIG. 2. The $(h k \ell)=(\overline{1} 00.3)$ reflection during Sb deposition at $250^{\circ} \mathrm{C}$. After $\frac{1}{3} \mathrm{ML}$ a $(\sqrt{3} \times \sqrt{3}) R 30^{\circ}$ reconstruction is formed. 
theoretical calculations [24] or recent experimental studies $[25,26]$ on this system so far.

Next, we determine the effect of $\mathrm{Sb}$ on the growth of Ag. Below RT predeposition of $\mathrm{Sb}$ has no significant effect on the formation of twin crystals, although the growth mode changes from 3D to layer-by-layer as reported previously [2]. At $100{ }^{\circ} \mathrm{C}$ no stacking faults occur for the clean surface, but predeposition of $0.3 \mathrm{ML}$ of $\mathrm{Sb}$ has a profound effect on the CTR intensity distribution. In Fig. 3(a) the peak intensity of the $(h k \ell)=(\overline{1} 00.3)$ reflection is shown during deposition at $100{ }^{\circ} \mathrm{C}$. Different stages of the growth are schematically depicted in Fig. 3(b). First the intensity decreases because of an increase in surface roughness due to the deposition of $\mathrm{Sb}$ (note the difference with the near equilibrium situation at $T=250{ }^{\circ} \mathrm{C}$ in Fig. 2). On starting the $\mathrm{Ag}$ deposition a large increase in the yield is observed. Thereafter, the intensity oscillates with a 1 ML period and decays slowly back to the starting level. The initial increase indicates that all of the $\mathrm{Ag}$ (and $\mathrm{Sb}$ ) atoms in the top layer occupy hcp sites. The increase of more than a factor of 5 is the same as that measured in the transition to the $\sqrt{3}$ reconstruction shown in Fig. 2. When the buried $\mathrm{Sb}$ atoms segregate towards the surface during deposition, all $\mathrm{Ag}$ atoms in lower levels return to energetically favored fcc sites. So the stacking fault is present only in the top layer

a)

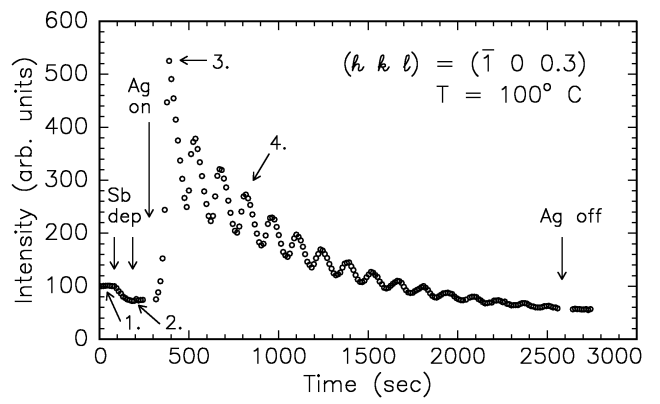

b)

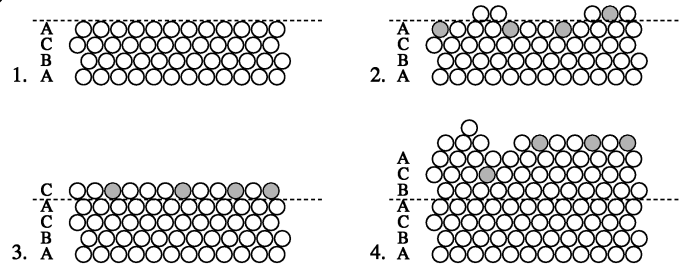

FIG. 3. (a) The $(h k \ell)=(\overline{1} 00.3)$ reflection during $0.3 \mathrm{ML}$ $\mathrm{Sb}$ deposition followed by $16 \mathrm{ML}$ of $\mathrm{Ag}$ at $100^{\circ} \mathrm{C}$. The numbers assign the different states depicted in (b). (b) Schematic side view of the surface during different stages of deposition. Open circles represent $\mathrm{Ag}$ atoms and filled circles represent $\mathrm{Sb}$ atoms. On the clean $\mathrm{Ag}(111)$ surface (1) $0.3 \mathrm{ML}$ of $\mathrm{Sb}$ is deposited. The $\mathrm{Sb}$ is either on top or embedded in the top surface layer (2). After a total deposition $(\mathrm{Sb}+\mathrm{Ag})$ of $1 \mathrm{ML}$ all atoms are on hcp sites (3). After $4 \mathrm{ML}$ deposition (4) the surface atoms are partly correctly stacked (left) and partly hcp stacked (right). The starting interface has returned to the correct fcc stacking $(A B C A b c a \ldots)$. and "floats" on the surface. Because the segregation of $\mathrm{Sb}$ is incomplete the total number of atoms at hcp sites slowly decreases during growth and results in the overall decrease in intensity. After $16 \mathrm{ML}$ we find that $20 \%$ of the surface area has a stacking fault. The intensity is also reduced by the increasing surface roughness. During similar depositions the growth was interrupted at various points to measure the full CTR, allowing a detailed evaluation of the surface atomic structure and roughness. From this we know that indeed only the top layer has the hcp stacking and that the buried layers have the normal fcc stacking. No twinning is observed. Note that without the floating stacking fault, the oscillation amplitude would not rise above the starting value.

Surprisingly, a predeposition of less than $\frac{1}{3} \mathrm{ML}$ of $\mathrm{Sb}$ has a similar effect, as illustrated in Fig. 4. A deposition at $100{ }^{\circ} \mathrm{C}$ of $0.2 \mathrm{ML} \mathrm{Sb}$ does not directly lead to a stacking fault in the top layer, as we have already concluded from the data in Figs. 2 and 3. When, however, we deposit on this surface $0.8 \mathrm{ML}$ of $\mathrm{Ag}$ all atoms in the top layer are wrongly stacked, while the absence of fractional order reflections indicates that no long range ordered $\sqrt{3}$ reconstruction exists. The solid curve in Fig. 4 shows the expected structure factor distribution for a top layer in which all the atoms are on hcp sites. For smaller amounts of $\mathrm{Sb}$ the top layer has only partly hcp stacking. This is illustrated for a deposition of 0.1 ML Sb followed by $5 \mathrm{ML}$ of Ag. The stacking fault is still clearly visible in the CTR. The dash-dotted curve is a calculation for a top layer of which $10 \%$ of the atoms are on hcp sites and the remainder on normal fcc positions. The stacking faults at

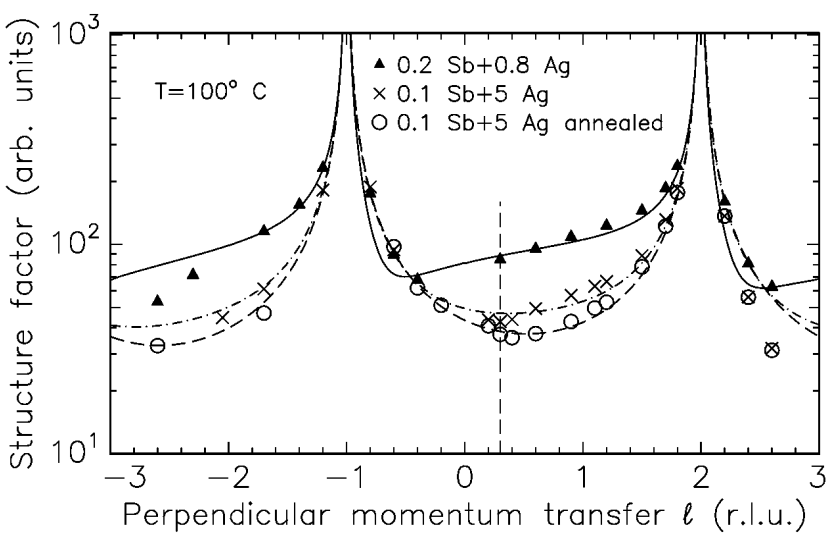

FIG. 4. Structure factors along the $(h k)=(\overline{1} 0)$ CTR at $100{ }^{\circ} \mathrm{C}$. After 0.2 ML Sb plus 0.8 ML Ag deposition all toplayer atoms are on hep sites (triangles). The solid curve represents a calculation for this. The crosses represent the data for a surface covered with $0.1 \mathrm{ML} \mathrm{Sb}$ and $5 \mathrm{ML} \mathrm{Ag}$, which was thereafter annealed (open circles). The dash-dotted curve is a calculation for a state where $10 \%$ of the top layer has the hcp stacking and the rest has the normal fcc stacking. The dashed curve is found for the bulk terminated clean $\operatorname{Ag}(111)$ (data not shown). The vertical dashed line marks the position

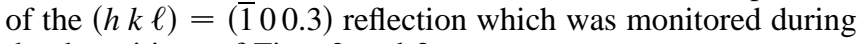
the depositions of Figs. 2 and 3. 
this coverage are formed only due to kinetic limitations. After annealing of the layer, the CTR profile is identical to that of the clean bulk terminated crystal (open circles), meaning that all atoms occupy normal fcc sites.

Next we explain these observations. STM experiments at RT [22,27] have shown that after deposition of small amounts of $\mathrm{Sb}$ on $\mathrm{Ag}(111), \mathrm{Sb}$ is present in two states: (1) as single embedded atoms in the top surface layer, and (2) incorporated in small islands with a $\sqrt{3}$ superstructure. It was shown that subsequently deposited $\mathrm{Ag}$ atoms attach to these $\sqrt{3}$ islands. From the data in Fig. 4 we conclude that these Ag atoms follow the hcp stacking of the $\sqrt{3}$ islands, even when the $\mathrm{Sb}$ coverage is less than $\frac{1}{3} \mathrm{ML}$. After the first $\mathrm{ML}$ is completed, all $\mathrm{Sb}$ is embedded in the top layer and no $\sqrt{3}$ islands are present on top $[22,28]$. Since we still observe some hcp stacking at higher coverage, a different mechanism must be active in these subsequent layers. This is provided by the fact that continued $\mathrm{Ag}$ growth leads to a place-exchange mechanism with embedded $\mathrm{Sb}$ [28]. If approximately one out of three arriving $\mathrm{Ag}$ atoms "releases" an $\mathrm{Sb}$ atom, the effective surface $\mathrm{Sb}$ concentration is $\frac{1}{3}$ and $\sqrt{3}$ islands can be nucleated. These newly formed islands then act as centers from which the hcp stacking propagates. Obviously, this is a statistical process. For a low $\mathrm{Sb}$ concentration, islands with the proper stacking will also occur. This picture is corroborated by our observation that when a surface covered with $0.2 \mathrm{ML}$ of $\mathrm{Sb}$ is annealed prior to Ag deposition, only $40 \%$ of the surface has a stacking fault after $1 \mathrm{ML}$ deposition (not shown), while without annealing the full top layer is wrongly stacked (see Fig. 4). In the latter case, there are already enough $\sqrt{3}$ islands on top of the surface, while for the annealed case these have to be produced by the exchange mechanism.

Upon continued growth the Ag atoms in the top layer return to fcc stacking positions, meaning that all atoms have to move from hcp to fcc sites. How this occurs is, at present, unclear. It seems likely that this process occurs at the edges of growing islands. A microscopic technique, like low-energy electron microscopy, is needed to clarify this issue. A similar registry shift of a single layer was reported recently for $\mathrm{Cu} / \mathrm{Co} / \mathrm{Cu}(111)$ sandwiches. It was found that thin cobalt films on $\mathrm{Cu}(111)$ can change from hcp stacking to fcc stacking when covered with a copper layer [29].

In summary, we have shown that $\mathrm{Sb}$ has a remarkable effect on the formation of stacking faults in the homoepitaxial growth of $\mathrm{Ag}(111)$. At an $\mathrm{Sb}$ coverage above $\frac{1}{3}$ ML, thermodynamic equilibrium is achieved by a top layer with hcp stacking and a $\sqrt{3}$ reconstruction. At such an Sb coverage, deposition of Ag leads to a floating stacking fault. However, due to kinetic limitations and due to an exchange process between $\mathrm{Sb}$ and $\mathrm{Ag}$ atoms, even for lower $\mathrm{Sb}$ coverages a floating stacking fault is formed during $\mathrm{Ag}$ growth. For growth above $100{ }^{\circ} \mathrm{C}$, all lower lying $\mathrm{Ag}$ layers return to the correct stacking, and no twin crystallites are formed.

This work is part of the research program of the Foundation for Fundamental Research on Matter (FOM) and was made possible by financial support from the Netherlands Organization for Scientific Research (NWO).

*Present address: University of Amsterdam, Van der Waals-Zeeman Institute, Valckenierstraat 65, 1018 XE Amsterdam, The Netherlands.

[1] B. Poelsema et al., Appl. Phys. A 53, 369 (1991).

[2] H. A. van der Vegt et al., Phys. Rev. Lett. 68, 3335 (1992).

[3] S. Esch, M. Hohage, Th. Michely, and G. Comsa, Phys. Rev. Lett. 72, 518 (1994).

[4] H. A. van der Vegt et al., Phys. Rev. B 52, 17443 (1995).

[5] A.W. Denier van der Gon, R. M. Tromp, and M.C. Reuter, Thin Solid Films 236, 140 (1993).

[6] K.-H. Park, J. S. Ha, S.-J. Park, and E.-H. Lee, Surf. Sci. 380, 258 (1997).

[7] T. Lewowski and P. Wieczorek, Surf. Rev. Lett. 4, 219 (1997).

[8] H. A. van der Vegt et al., Surf. Sci. 365, 205 (1996).

[9] J. Camarero et al., Phys. Rev. Lett. 73, 2448 (1994).

[10] J. J. de Miguel, Surf. Rev. Lett. 4, 353 (1997).

[11] K. Meinel, M. Klaua, and H. Bethge, Phys. Status Solidi (a) 110, 189 (1988).

[12] Ch. Ammer et al., Surf. Sci. 307-309, 570 (1994).

[13] E.Z. Luo, J. Wollschläger, F. Wegner, and M. Henzler, Appl. Phys. A 60, 19 (1995).

[14] C. Ratsch, A. P. Seitsonen, and M. Scheffler, Phys. Rev. B 55, 6750 (1997).

[15] I. K. Robinson, Phys. Rev. B 33, 3830 (1986).

[16] I. K. Robinson and D. J. Tweet, Rep. Prog. Phys. 55, 599 (1992).

[17] C. Norris et al., Rev. Sci. Instrum. 63, 1083 (1992).

[18] E. Vlieg et al., Nucl. Instrum. Methods Phys. Res., Sect. A 262, 522 (1987).

[19] M. Lohmeier and E. Vlieg, J. Appl. Crystallogr. 26, 706 (1993).

[20] E. Vlieg, J. Appl. Crystallogr. 30, 532 (1997).

[21] Apparently, the surface is less rough than measured during specular reflectivity [2]. This is probably due to the fact that only $2 \mathrm{ML}$ were deposited and that some annealing has occurred.

[22] H. A. van der Vegt, J. Vrijmoeth, R. J. Behm, and E. Vlieg, Phys. Rev. B 57, 4127 (1998).

[23] S. A. de Vries et al., Surf. Sci. (to be published).

[24] S. Oppo, V. Fiorentini, and M. Scheffler, Phys. Rev. Lett. 71, 2437 (1993).

[25] T. C. Q. Noakes, D. A. Hutt, C.F. McConville, and D. P. Woodruff, Surf. Sci. 372, 117 (1997).

[26] H. Cruguel et al., Phys. Rev. B 55, R16061 (1997).

[27] J. Vrijmoeth et al., Phys. Rev. Lett. 72, 3843 (1994).

[28] J. A. Meyer et al., Surf. Sci. 355, L375 (1996).

[29] Ch. Rath et al., Phys. Rev. B 55, 10791 (1997). 